Internat. J. Math. \& Math. Sci.

Vol. 22, No. 1 (1999) 205-208

S 0161-1712<99>22205-3

(c) Electronic Publishing House

\title{
TOTALLY REAL SUBMANIFOLDS IN A COMPLEX PROJECTIVE SPACE
}

\author{
LIU XIMIN
}

(Received 23 July 1996 and in revised form 13 December 1996)

\begin{abstract}
In this paper, we establish the following result: Let $M$ be an $n$-dimensional complete totally real minimal submanifold immersed in $C P^{n}$ with Ricci curvature bounded from below. Then either $M$ is totally geodesic or $\inf r \leq(3 n+1)(n-2) / 3$, where $r$ is the scalar curvature of $M$.
\end{abstract}

Keywords and phrases. Complex projective space, totally real submanifold, Ricci curvature.

1991 Mathematics Subject Classification. 53C40, 53C55.

1. Introduction. Let $C P^{n}$ be the $n$-dimensional complex projective space with the Fubini-Study metric of constant holomorphic sectional curvature $c=4$ and let $M$ be an $n$-dimensional totally real submanifold of $C P^{n}$. Let $r$ be the scalar curvature of $M$. If $M$ is compact, then many authors studied them and obtained many beautiful results (for example [2, 4, 5]).

In this paper, we make use of Yau's maximum principle to study the complete totally real minimal submanifold with Ricci curvature bounded from below and obtain the following result.

THEOREM 1. Let $M$ be an n-dimensional complete totally real minimal manifold immersed in $C P^{n}$ with Ricci curvature bounded from below. Then either $M$ is totally geodesic or $\inf r \leq(3 n+1)(n-2) / 3$.

2. Preliminaries. Let $M$ be an $n$-dimensional totally real minimal submanifold of $C P^{n}$. We choose a local field of orthonormal frames $e_{1}, \ldots, e_{n}, e_{1^{*}}=J e_{1}, \ldots, e_{n^{*}}=J e_{n}$ ( $J$ is the complex structure of $C P^{n}$ ), such that, restricted to $M$, the vectors $e_{1}, \ldots, e_{n}$ are tangent to $M$. We make use of the following convention on the range of indices

$$
A, B, C, \ldots=1, \ldots, n, 1^{*}, \ldots, n^{*} ; \quad i, j, k, \ldots=1, \ldots, n .
$$

With respect to the frame field of $C P^{n}$, let $w^{A}$ be the field of dual frames. Then the structure equations of $C P^{n}$ are given by

$$
\begin{aligned}
& d w^{A}=-\sum w_{B}^{A} \wedge w^{B}, \quad w_{A}^{B}+w_{B}^{A}=0, \\
& d w_{B}^{A}=-\sum w_{C}^{A} \wedge w_{B}^{C}+\frac{1}{2} \sum \bar{R}_{B C D}^{A} w^{C} \wedge w^{D}, \\
& \bar{R}_{B C D}^{A}=\delta_{A C} \delta_{B D}-\delta_{A D} \delta_{B C}+J_{A C} J_{B D}-J_{A D} J_{B C}+2 J_{A B} J_{C D},
\end{aligned}
$$


where $J=J_{A B} e_{A} \otimes e_{B}$, so that

$$
\left(J_{A B}\right)=\left(\begin{array}{cc}
0 & -I_{n} \\
I_{n} & 0
\end{array}\right),
$$

where $I_{n}$ is the identity matrix of order $n$. We restrict these forms to $M$. Then from [2], we have

$$
\begin{aligned}
w^{i^{*}} & =0, \quad w_{i}^{j}=w_{i^{*}}^{j^{*}}, \quad w_{j}^{i^{*}}=w_{i}^{j^{*}}, \\
w_{i}^{k^{*}} & =\sum h_{i j}^{k^{*}} w^{j}, \quad h_{i j}^{k^{*}}=h_{j i}^{k^{*}}=h_{j k}^{i^{*}}=h_{i k}^{j^{*}}, \\
d w^{i} & =-\sum w_{j}^{i} \wedge w^{j}, \quad w_{i}^{j}+w_{j}^{i}=0, \\
d w_{i}^{j} & =-\sum w_{i}^{k} \wedge w_{k}^{j}+\frac{1}{2} \sum R_{i k l}^{j} w^{k} \wedge w^{l}, \\
R_{j k l}^{i} & =\bar{R}_{j k l}^{i} w^{k}+\sum\left(h_{i k}^{m^{*}} h_{j l}^{m^{*}}-h_{i l}^{m^{*}} h_{j k}^{m^{*}}\right), \\
d w_{j^{*}}^{i^{*}} & =-\sum w_{k^{*}}^{i^{*}} \wedge w_{j^{*}}^{k^{*}}+\frac{1}{2} \sum R_{j^{*} k l}^{i^{*}} w^{k} \wedge w^{l}, \\
R_{j^{*} k l}^{i^{*}} & =\bar{R}_{j^{*} k l}^{i^{*}}+\sum\left(h_{k m}^{i^{*}} h_{m l}^{j^{*}}-h_{m l}^{i^{*}} h_{k m}^{j^{*}}\right) .
\end{aligned}
$$

The second fundamental form $h$ of $M$ in $C P^{n}$ is defined as $h=\sum h_{i j}^{k^{*}} w^{i} \otimes e_{k^{*}}$, whose squared length is $\|h\|^{2}=\sum\left(h_{i j}^{k^{*}}\right)^{2}$.

If $M$ is minimal in $C P^{n}$, i.e., trace $h=0$, then from (2.4) and (2.10), we have

$$
r=n(n-1)-\|h\|^{2},
$$

where $r$ is the scalar curvature of $M$.

Define $h_{i j k}^{m^{*}}$ and $h_{i j k l}^{m^{*}}$ by

$$
\begin{aligned}
& \sum h_{i j k}^{m^{*}} w^{k}=d h_{i j}^{m^{*}}-\sum h_{k j}^{m^{*}} w_{i}^{k}-\sum h_{i k}^{m^{*}} w_{j}^{k}+\sum h_{i j}^{l^{*}} w_{l^{*}}^{m^{*}}, \\
& \sum h_{i j k l}^{m^{*}} w^{l}=d h_{i j k}^{m^{*}}-\sum h_{l j k}^{m^{*}} w_{i}^{l}-\sum h_{i l k}^{m^{*}} w_{j}^{l}-\sum h_{i j l}^{m^{*}} w_{k}^{l}+\sum h_{i j k}^{l^{*}} w_{l^{*}}^{m^{*}},
\end{aligned}
$$

respectively.

Let $H_{l^{*}}$ and $\Delta$ denote the $(n \times n)$-matrix $\left(h_{i j}^{l^{*}}\right)$ and the Laplacian on $M$, respectively. By a simple calculation, we have (cf. [2])

$$
\begin{aligned}
\frac{1}{2} \Delta\|h\|^{2}= & \sum\left(h_{i j k}^{l^{*}}\right)^{2}+(n+1)\|h\|^{2}+\sum \operatorname{tr}\left(H_{i^{*}} H_{j^{*}}-H_{j^{*}} H_{i^{*}}\right)^{2} \\
& -\sum\left(\operatorname{tr} H_{i^{*}} \operatorname{tr} H_{j^{*}}\right)^{2} .
\end{aligned}
$$

The following lemma is important in this paper.

LEMMA 1 [6]. Let $M^{n}$ be a complete Riemannian manifold with Ricci curvature bounded from below and let $f$ be a $C^{2}$-function bounded from above on $M^{n}$, then for all $\epsilon>0$, there exists a point $x \in M^{n}$ at which
(i) $\sup f-\epsilon<f(x)$;
(ii) $\|\nabla f(x)\|<\epsilon$;
(iii) $\Delta f(x)<\epsilon$. 
Proof OF THE MAIN THEOREM. By [3], we have $\sum\left(\operatorname{tr} H_{i^{*}} H_{j^{*}}\right)^{2}=\sum\left(\operatorname{tr} H_{i^{*}}^{2}\right)^{2}$. From [1], we know that $\sum \operatorname{tr}\left(H_{i^{*}} H_{j^{*}}-H_{j^{*}} H_{i^{*}}\right)^{2}-\sum\left(\operatorname{tr} H_{i^{*}}^{2}\right)^{2} \geq-3 / 2\|h\|^{4}$. So, from (2.16), we obtain

$$
\frac{1}{2} \Delta\|h\|^{2} \geq\|h\|^{2}\left((n+1)-3 / 2\|h\|^{2}\right) .
$$

We know that $\|h\|^{2}=n(n-1)-r$. By the condition of the theorem, we conclude that $\|h\|^{2}$ is bounded. We define $f=\|h\|^{2}$ and $F=(f+a)^{1 / 2}$ (where $a>0$ is any positive constant number). $F$ is bounded. We have

$$
\begin{aligned}
d F & =\frac{1}{2}(f+a)^{-1 / 2} d f, \\
\Delta F & =\frac{1}{2}\left(-\frac{1}{2}(f+a)^{-3 / 2}\|d f\|^{2}+(f+a)^{-1 / 2} \Delta f\right) \\
& =\frac{1}{2}\left(-2\|d F\|^{2}+\Delta f\right)(f+a)^{-1 / 2},
\end{aligned}
$$

i.e.,

$$
\Delta F=\frac{1}{2 F}\left(-2\|d F\|^{2}+\Delta f\right) .
$$

Hence, $F \Delta F=-\|d F\|^{2}+1 / 2 \Delta f$ or $1 / 2 \Delta f=F \Delta F+\|d F\|^{2}$.

Applying Lemma 1 to $F$, we have for all $\epsilon>0$, there exists a point $x \in M$ such that at $x$

$$
\begin{gathered}
\|d F(x)<\epsilon\| ; \\
\Delta F(x)<\epsilon ; \\
F(x)>\sup F-\epsilon .
\end{gathered}
$$

From (2.21), (2.22), and (2.23), we have

$$
\frac{1}{2} \Delta f<\epsilon^{2}+F \epsilon=\epsilon(\epsilon+F)
$$

We take a sequence $\left\{\epsilon_{m}\right\}$ such that $\epsilon_{m} \longrightarrow 0(m \longrightarrow \infty)$ and for all $m$, there exists a point $x_{m} \in M$ such that (2.21), (2.22), and (2.23) hold. Therefore, $\epsilon_{m}\left(\epsilon_{m}+F\left(x_{m}\right)\right) \longrightarrow$ $0(m \rightarrow \infty)$ (because $F$ is bounded).

From (2.23), we have $F\left(x_{m}\right)>\sup F-\epsilon_{m}$. Because $\left\{F\left(x_{m}\right)\right\}$ is a bounded sequence. So we get $F\left(x_{m}\right) \longrightarrow F_{0}$ (if necessary, we can choose a subsequence). Hence, $F_{0} \geq \sup F$. So we have

$$
F_{0}=\sup F
$$

From the definition of $F$, we get

$$
f\left(x_{m}\right) \rightarrow f=\sup f .
$$

(2.17) and (2.24) imply that

$$
f\left((n+1)-\frac{3}{2} f\right) \leq \frac{1}{2} \Delta f \leq \epsilon(\epsilon+F),
$$

and

$$
f\left(x_{m}\right)\left((n+1)-\frac{3}{2} f\left(x_{m}\right)\right)<\epsilon_{m}^{2}+\epsilon_{m} F\left(x_{m}\right) \leq \epsilon_{m}^{2}+\epsilon_{m} F_{0}
$$


let $m \longrightarrow \infty$, then $\epsilon_{m} \longrightarrow 0$ and $f\left(x_{m}\right) \longrightarrow f_{0}$. Hence,

$$
f_{0}\left((n+1)-\frac{3}{2} f_{0}\right) \leq 0 .
$$

(i) if $f_{0}=0$, we have $f=\|h\|^{2} \equiv 0$. Hence, $M$ is totally geodesic.

(ii) if $f_{0}>0$, we have $(n+1)-3 / 2 f_{0} \leq 0$ and $f_{0} \geq 2 / 3(n+1)$, that is, sup $\|h\|^{2} \geq$ $2 / 3(n+1)$. Therefore, $\inf r \leq(3 n+1)(n-2) / 3$. This completes the proof.

ACKNOWLEDGEMENT. The author is grateful to the referee for the careful reading and the very helpful comments on the earlier versions of this manuscript.

\section{REFERENCES}

[1] L. An Min and L. Jimin, An intrinsic rigidity theorem for minimal submanifolds in a sphere, Arch. Math. (Basel) 58 (1992), no. 6, 582-594. MR 93b:53050. Zbl 767.53042.

[2] B. Y. Chen and K. Ogiue, On totally real submanifolds, Trans. Amer. Math. Soc. 193 (1974), 257-266. MR 49 11433. Zbl 286.53019.

[3] S. S. Chern, M. do Carmo, and S. Kobayashi, Minimal submanifolds of a sphere with second fundamental form of constant length, Functional Analysis and Related Fields (Proc. Conf. for M. Stone, Univ. Chicago, 1968) (New York), vol. Ill, Springer-Verlag, 1970, pp. 59-75. MR 42\#8424. Zbl 216.44001.

[4] G. D. Ludden, M. Okumura, and K. Yano, A totally real surface in $C P^{2}$ that is not totally geodesic, Proc. Amer. Math. Soc. 53 (1975), no. 1, 186-190. MR 52 1580. Zbl 312.53043.

[5] Y. B. Shen, Scalar curvature for totally real minimal submanifolds, Chinese Ann. Math. Ser. A 12 (1991), no. 5, 573-577 (Chinese). MR 93a:53052. Zbl 777.53055.

[6] S. T. Yau, Harmonic functions on complete Riemannian manifolds, Comm. Pure Appl. Math. 28 (1975), 201-228. MR 55 4042. Zbl 297.31005.

Ximin: DePARTMENT OF MATHEMATICS, NANKAI UNIVERSITy, TIANJIN 300071, ChinA

Current address: DEPARTMENT OF APPLIED MATHEMATICS, DALIAN UNIVERSITY OF TECHNOLOGY, DALIAN 116024, CHINA

E-mail address: xm7iu@d7ut.edu.cn 


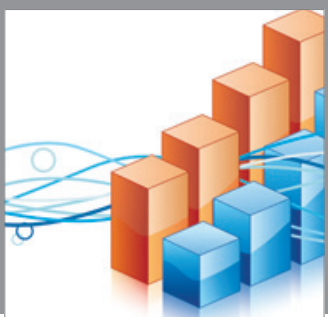

Advances in

Operations Research

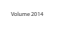

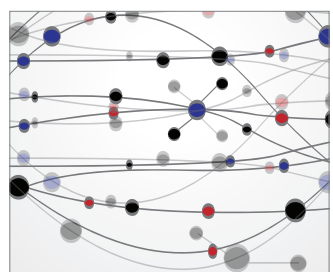

\section{The Scientific} World Journal
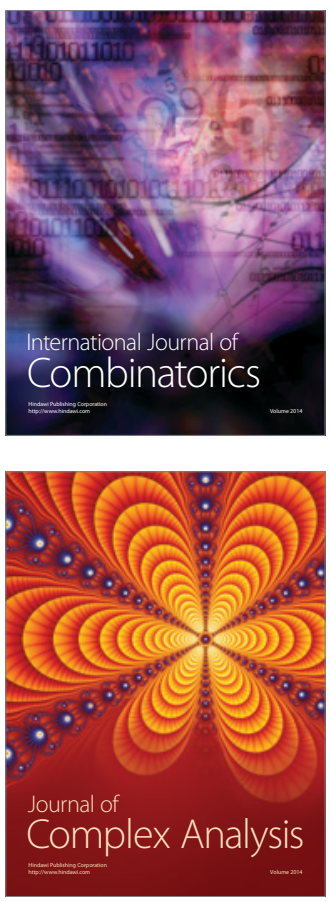

International Journal of

Mathematics and

Mathematical

Sciences
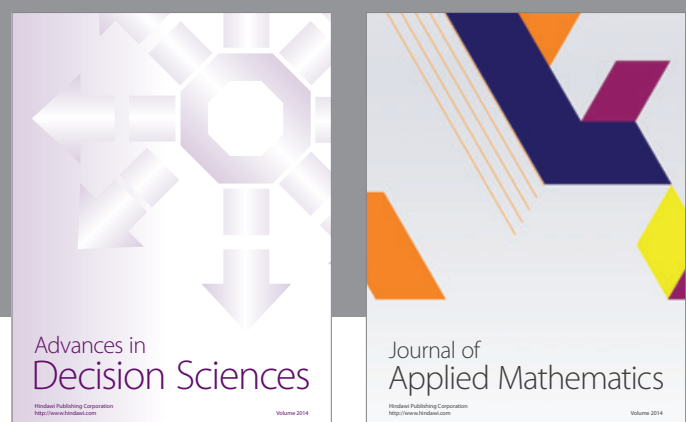

Journal of

Applied Mathematics
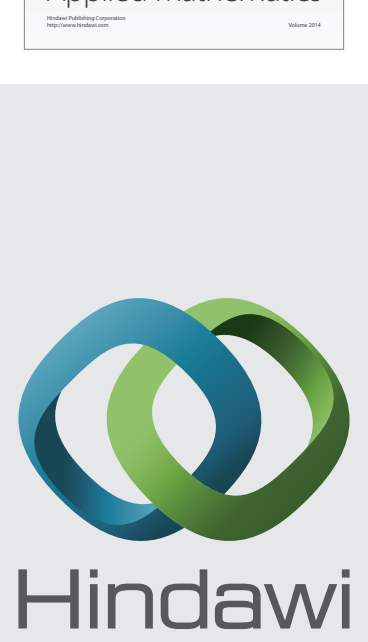

Submit your manuscripts at http://www.hindawi.com
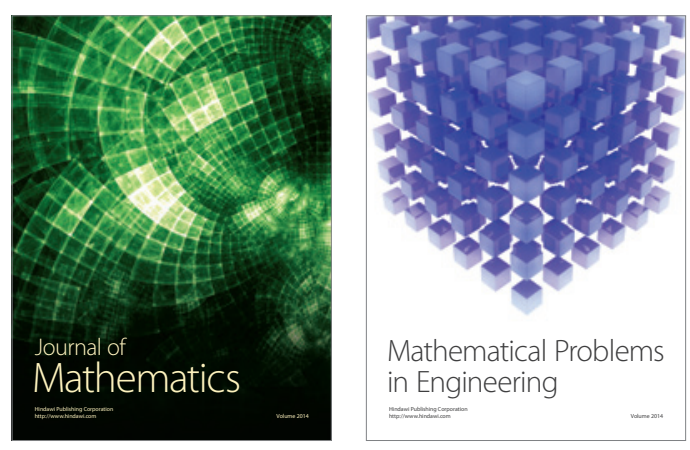

Mathematical Problems in Engineering
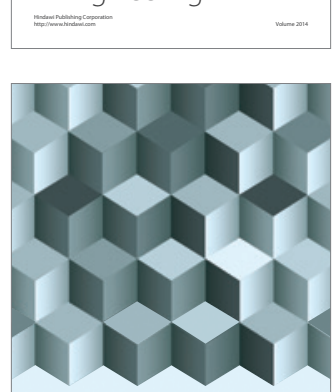

Journal of

Function Spaces
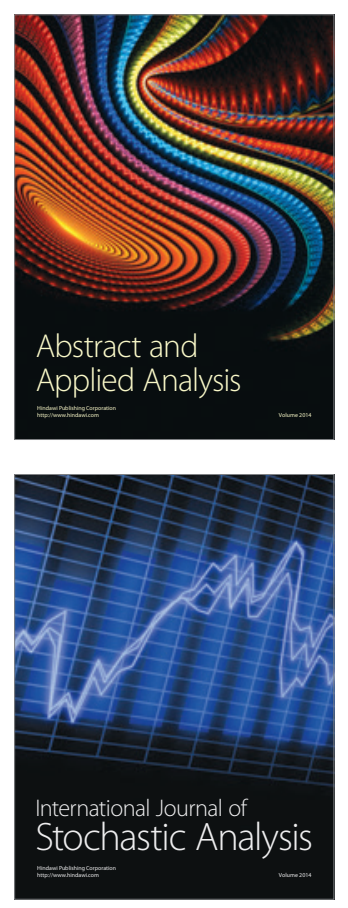

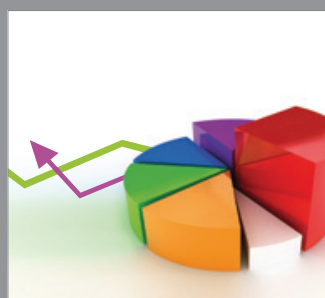

ournal of

Probability and Statistics

Promensencen
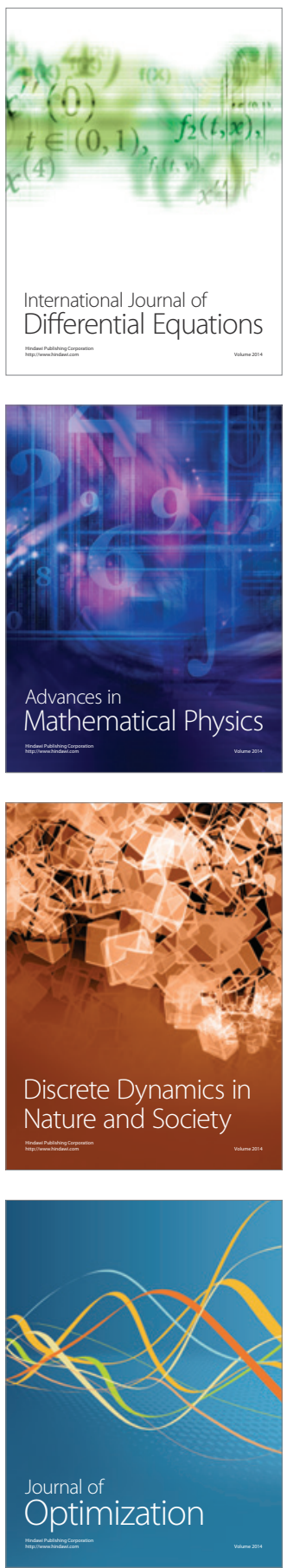